\title{
Transient hyperoxia and cerebral blood flow velocity in infants born prematurely and at full term
}

\author{
S NIIJIMA, D B SHORTLAND, M I LEVENE, AND D H EVANS \\ Neonatal Unit, Department of Child Health, Leicester University School of Medicine, Leicester
}

SUMmARY Little is known about the effects of hyperoxia on the cerebral circulation of human infants. Using duplex Doppler we measured the changes in cerebral blood flow velocity in a group of full term $(n=15)$ and premature infants $(n=17$, median gestational age 31 weeks) in response to a transient threefold increase in oxygen tension. Measurements of blood gas tensions as well as blood pressure and cerebral blood flow velocity were made over a period of 20 minutes on three occasions for each infant; during normal oxygenation, hyperoxia, and normal oxygenation. There was a fall in cerebral blood flow velocity in 15 of the 17 premature infants with hyperoxia and the median reduction was $0.06 \mathrm{~cm} /$ second for every $1 \mathrm{kPa}$ increase in oxygen tension. There was no significant change in either $\mathrm{PCO}_{2}$ or blood pressure during the period of hyperoxia. The cerebral blood flow velocity fell in all 15 infants born at full term during hyperoxia, but there was a simultaneous and significant reduction in $\mathrm{PCO}_{2}$ at the same time as the hyperoxia. Analysis of variance suggested that in the infants born at full term the change in carbon dioxide had most effect in the reduction of cerebral blood flow velocity, rather than the hyperoxia itself. We conclude that in premature infants, cerebral vascular resistance may be altered by a fall in cerebral blood flow velocity in the presence of hyperoxia.

Hyperoxia has a consistent and predictable effect on cerebral blood flow in healthy adult brains. Little is known, however, about the effect of hyperoxia on cerebral blood flow in newborn infants, although oxygen is commonly used to treat lung disease in this population. Periods of prolonged hyperoxia cause vasoconstriction of the neonatal retinal circulation (which arises from the internal carotid artery) and so would also be expected to have a similar effect on the cerebral circulation. Whether the central nervous system of the extremely premature newborn infant is also sensitive to the effects of hyperoxia is unknown.

We report the use of duplex Doppler ultrasound to study the physiological effect of extremely short periods of hyperoxia on the cerebral circulation in healthy and ill infants born prematurely and at full term.

\section{Methods}

Infants enrolled in this study were all born in the maternity unit of the Leicester Royal Infirmary and were selected if they had a normal cerebral ultrasound examination, no serious congenital abnormalities, and no clinical evidence of intrapartum asphyxia. Infants on mechanical ventilators were recovering from their lung disease, and arterial blood gas sampling was performed only through indwelling aortic or radial cannulas.

The infant's gestational age was calculated from the date of the mother's last menstrual period, and if there was any doubt about it a Dubowitz assessment of gestation was performed. ${ }^{1}$ There were three groups of infants: those receiving mechanical ventilation with extra oxygen, those requiring oxygen by headbox, and healthy mature infants born at full term with no lung disease.

Measurement of cerebral blood flow velocity was made with an ATL duplex Doppler mark 600 machine. The transducer (724A) contained a 7.5 $\mathrm{MHz}$ pulse/echo imaging crystal and a $5 \mathrm{MHz}$ pulse/echo Doppler crystal. At least 20 consecutive cardiac impulses were recorded onto audiotape and subsequently analysed using the computer system described by Schlindwein et al. ${ }^{2}$ This system .performs fast Fourier transform on the audio signal to produce Doppler power spectra every $6 \cdot 25 \mathrm{~ms}$. A maximum frequency envelope is then extracted from the spectral data using a threshold technique. ${ }^{3}$ 
The frequency measurements are converted to velocity measurements with the standard Doppler equation, and the mean velocity over the cardiac cycle is calculated by halving the mean measurement of the maximum velocity over the cardiac cycle. ${ }^{4}$

Before the study a transcutaneous oxygen or combined carbon dioxide and oxygen monitor was calibrated and applied to the anterior abdominal wall of the infant. Mean arterial blood pressure was measured by a Dinamap blood pressure monitor or directly from an arterial line where one was available. Each baby was studied under three conditions consecutively: normal oxygenation, hyperoxia, and normal oxygenation. After the transcutaneous monitor had stabilised, a measurement of cerebral blood flow velocity was made from the ascending portion of the anterior cerebral artery as previously described. ${ }^{5}$ The amount of oxygen inspired was then increased to produce a measured arterial oxygen tension of about three times the resting measurement. The infant was allowed to stabilise for five minutes and then further recordings of cerebral blood flow velocity, transcutaneous $\mathrm{PO}_{2}$, mean arterial blood pressure, and $\mathrm{PaCO}_{2}$ or transcutaneous $\mathrm{PCO}_{2}$ where appropriate, were made. The period of hyperoxia never exceeded 10 minutes. The third assessment of cerebral blood flow velocity, blood pressure, and blood gases was made five to 10 minutes after the concentration of inspired oxygen had been returned to the baseline measurement.

Statistical analyses were by the Wilcoxon paired test, the two tailed Mann-Whitney test, and analysis of variance.

The study was approved by the Leicestershire district ethical committee and infants were enrolled only with the full consent of their parents; the study was usually performed in the presence of the baby's mother.

\section{Results}

A total of 32 infants was studied, 17 were premature ( 36 weeks' gestation or less) and 15 born at full term (37-42 weeks' gestation). The median gestational age of the infants born at full term was 39 weeks and that of the premature group 31 (range 25-36). The median age at the time of the study was 2 days in the premature group and 3 days in the group born at full term (tables 1 and 2).

There were significant falls in cerebral blood flow velocity in 15 of the 17 premature infants with hyperoxia $(p=0.001)$ (fig 1$)$ and in all 15 of those born at full term $(p=0.0006)$ (fig 2$)$. The change in velocity was corrected for a standard rise of $1 \mathrm{kPa}$ in $\mathrm{PaO}_{2}$. The median reduction in cerebral blood flow velocity for every increase in oxygen tension of $1 \mathrm{kPa}$ was $0.06 \mathrm{~cm} / \mathrm{second}$ for premature infants and $0.18 \mathrm{~cm} / \mathrm{second}$ for those born at full term; this difference was significant $(p=0.0001)$.

The median changes in velocity for the normoxic, hyperoxic, and normoxic periods of the study for the two groups are shown in fig 3. There was no difference between the two normoxic periods for the infants born at full term, but in the group of premature infants the velocity remained significantly lower in the second normoxic period compared with the first $(p=0 \cdot 016)$. The median time between the second and third assessments in the premature group was 10 minutes.

Table 1 Details of 17 premature babies with hyperoxia

\begin{tabular}{llcll}
\hline $\begin{array}{l}\text { Case } \\
\text { No }\end{array}$ & $\begin{array}{l}\text { Gestational } \\
\text { age (weeks) }\end{array}$ & $\begin{array}{l}\text { Birth weight } \\
(\mathrm{g})\end{array}$ & $\begin{array}{l}\text { Age at } \\
\text { study } \\
\text { (hours) }\end{array}$ & $\begin{array}{l}\text { Intermittent } \\
\text { positive pressure } \\
\text { ventilation used }\end{array}$ \\
\hline 1 & 33 & 1300 & 6 & No \\
2 & 25 & 820 & 72 & Yes \\
3 & 32 & 1930 & 48 & No \\
4 & 29 & 1270 & 13 & Yes \\
5 & 29 & 1430 & 192 & Yes \\
6 & 29 & 1660 & 35 & Yes \\
7 & 36 & 2550 & 16 & Yes \\
8 & 34 & 2220 & $6 \cdot 5$ & Yes \\
9 & 33 & 2320 & 168 & Yes \\
10 & 35 & 1920 & 48 & Yes \\
11 & 31 & 1540 & 48 & No \\
12 & 34 & 1860 & 48 & No \\
13 & 34 & 1890 & 48 & No \\
14 & 26 & 1030 & 96 & No \\
15 & 32 & 2070 & 15 & Yes \\
16 & 30 & 740 & 30 & No \\
17 & 32 & 1390 & 96 & Yes \\
\hline
\end{tabular}

Table 2 Details of 15 full term infants with hyperoxia

\begin{tabular}{lllll}
\hline $\begin{array}{l}\text { Case } \\
\text { No }\end{array}$ & $\begin{array}{l}\text { Gestational } \\
\text { age (weeks) }\end{array}$ & $\begin{array}{l}\text { Birth weight } \\
(g)\end{array}$ & $\begin{array}{l}\text { Age at study } \\
\text { (hours) }\end{array}$ & $\begin{array}{l}\text { Intermittent } \\
\text { positive pressure } \\
\text { ventilation used }\end{array}$ \\
\hline 1 & 40 & 3370 & 120 & No \\
2 & 41 & 3560 & 48 & No \\
3 & 37 & 2190 & 24 & No \\
4 & 39 & 3130 & 48 & Yes \\
5 & 38 & 3800 & 29 & Yes \\
6 & 42 & 3390 & 18 & Yes \\
7 & 40 & 3050 & 120 & No \\
8 & 39 & 2940 & 96 & No \\
9 & 37 & 3090 & 20 & Yes \\
10 & 40 & 3150 & 240 & No \\
11 & 39 & 4140 & 37 & No \\
12 & 37 & 3470 & 196 & No \\
13 & 39 & 3720 & 72 & No \\
14 & 38 & 3430 & 96 & No \\
15 & 39 & 2720 & 9 & Yes \\
\hline
\end{tabular}




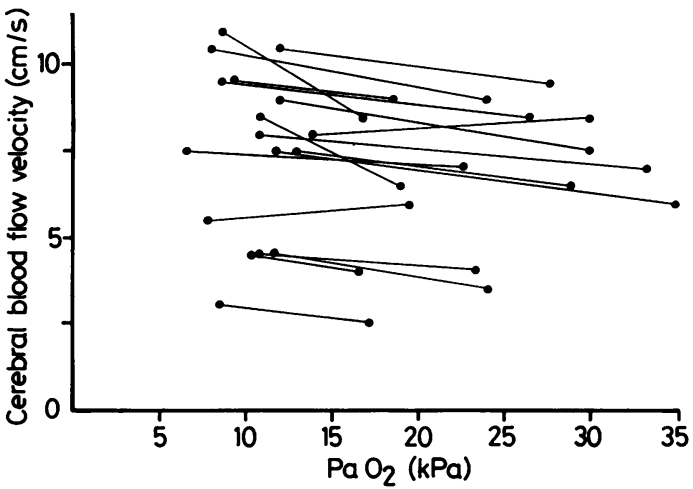

Fig 1 Change in cerebral blood flow velocity induced by increasing oxygen tension in infants of less than 37 weeks' gestation $(p=0.0012)$.

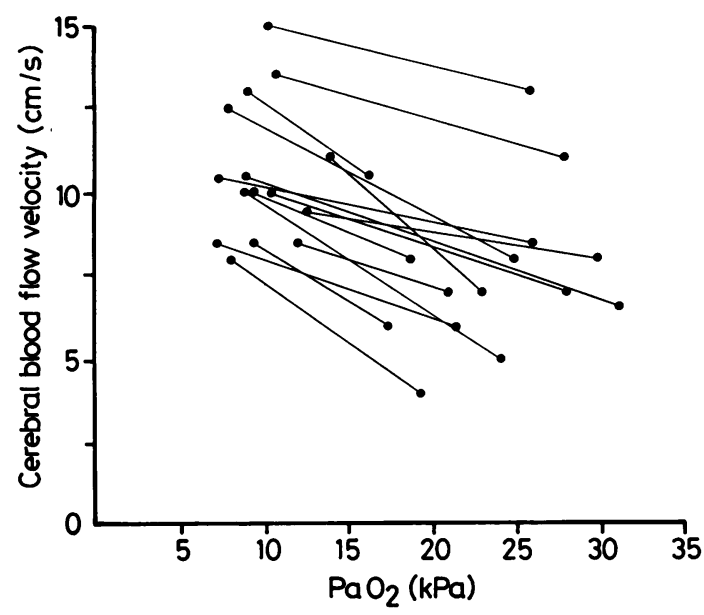

Fig 2 Change in cerebral blood flow velocity induced by increasing oxygen tension in infants of 37-42 weeks' gestation $(p=0.006)$.

There were no significant changes in the mean arterial blood pressure during the three assessment periods for either group. In the premature group carbon dioxide tension measurements were available in 13 of the 17 infants and there were no significant differences among the three assessment periods. Carbon dioxide tension measurements were available in 11 of the 15 infants born at full term, and in this group the median carbon dioxide tension for the normoxic, hyperoxic, and normoxic groups were $5 \cdot 1,4 \cdot 7$, and $5.0 \mathrm{kPa}$. When the two periods of normal oxygenation were compared with the hyperoxic period, there was a significant reduction in carbon dioxide tension with rising oxygen (the first normal period compared with the hyperoxic period $p=0.007$, and the hyperoxic period

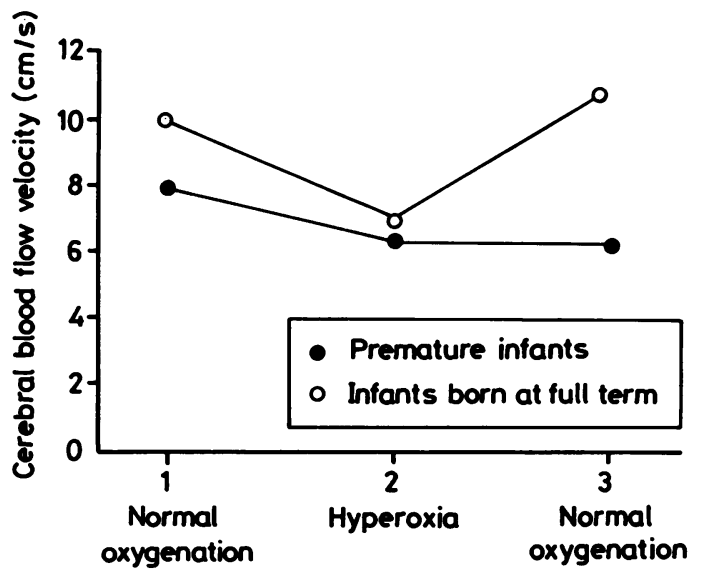

Fig 3 Median changes in cerebral blood flow velocity at the three assessments.

compared with the second normal period $\mathrm{p}=0.05$ ). Analysis of variance showed that a change in oxygen tension contributed $1.3 \%$ of the variability in cerebral blood flow velocity compared with $10.6 \%$ that was attributable to changes induced by alterations in the carbon dioxide tension.

\section{Discussion}

Our study shows that newborn infants undergo physiological changes in their cerebral blood flow velocity with hyperoxia, and this effect was present during the first few days of life. We previously reported that extremely ill, premature infants undergo an increase in their cerebral blood flow velocity with a small increase in arterial carbon dioxide tension. ${ }^{6}$ These studies show that in newborn infants irrespective of gestational age changes can be mediated in cerebral blood flow by altering blood gas tensions in the same manner as in adults.

Hypoxia increases cerebral blood flow in fetal $^{7}$ and newborn ${ }^{8}$ lambs. Rosenberg $e a^{8} l^{8}$ also showed that the increase in cerebral blood flow (measured by microspheres and induced by hypoxia) correlated closely with changes in both diastolic velocity and peak systolic velocities (measured by Doppler ultrasound). Costeloe et al showed a reduction in apparent cerebral blood flow with hyperoxia using indirect methods of measuring cerebral blood flow such as venous occlusion plethysmography and electrical impedance, but the infants that they studied were more mature than the ones we report.

There has been some suggestion ${ }^{10}$ (based on the work of Mchedlishvili ${ }^{11}$ ) that Doppler ultrasound may not reliably reflect changes in cerebral blood 
flow caused by the changing diameter of the vessel being examined (in this case the anterior cerebral artery). If, however, hyperoxia did induce vasoconstriction of the anterior cerebral artery, this effect would only underestimate any change detected by Doppler ultrasound. Mchedlishvili recently concluded that changes in cerebral blood flow caused by changes in oxygen or carbon dioxide tensions are mainly mediated through the small pial arteries. ${ }^{12}$ We believe that changes in cerebral blood flow velocity measured by duplex Doppler ultrasound reflect actual changes in cerebral blood flow caused by the resistance of the cerebral microvasculature.

The infants born at full term seemed to have greater reduction in cerebral blood flow velocity than the premature ones. This effect was principally caused by significant falls in carbon dioxide tensions during the periods of hyperoxia. Increasing inspired oxygen to the mature spontaneously breathing newborn infant probably stimulated respiration with a consequent reduction in carbon dioxide tension. This may have been mediated peripherally by the flow of gas close to the babies' faces. An alternative hypothesis is that the reduction in pulmonary vascular resistance increased the pulmonary blood flow and hence reduced the $\mathrm{PaCO}_{2}$. We cannot be sure whether hyperoxia alone changes the cerebral blood flow velocity in the mature babies we studied, because of the comparatively greater effect of the change in carbon dioxide tension. Most of the premature infants we studied were mechanically ventilated and consequently did not show systematic changes in their carbon dioxide tensions. Hypoxia and hypercapnia have additive effects in increasing cerebral blood flow ${ }^{13}$ and we might assume that hyperoxia and hypocapnia have similar additive effects.

Another interesting difference between premature infants and those born at full term was the failure of the premature infants' cerebral blood flow velocity to return to normal after periods of hyperoxia (fig 3). This may be because premature infants have less efficient control of their cerebral arterioles, and there is a delay in vasodilatation and return to normally oxygenated cerebral blood flow. We may also be observing a hysteresis loop with proportionately less reduction in cerebral blood flow velocity in the downward part of the oxygen tension curve. With the introduction of more continuous methods for measuring cerebral blood flow velocity we may be able to study this in more detail.

Infants born at full term (and possibly also premature infants) with sustained degrees of hyperoxia are more likely to develop retinopathy of prematurity and this is presumably due to a period of retinal ischaemia. The retinal arterioles are not likely to be any more sensitive to hyperoxia than other arterioles within the central nervous system, and our results show that there is a consistent reduction in cerebral blood flow velocity (and therefore cerebral blood flow) with a short period of hyperoxia in the immature brain itself. It seems possible therefore, that the effects of a short period of hyperoxia on the brain of an extremely premature infant may be more prolonged than on the brain of a baby born at full term, and that this may be an important factor in the development of retinopathy of prematurity in these infants. We believed that it was justified and safe to expose these infants (most of whom were receiving oxygen anyway) to an extremely short period of hyperoxia. Regular ophthalmic examinations of the premature infants were performed and in no case where acute retinopathy of prematurity occurred was it more severe than grade II.

In conclusion, the cerebral blood flow velocity of the newborn infant alters in response to a brief period of hyperoxia. In the premature infant this effect seems to be more prolonged than that in the infant born at full term. The exaggerated reduction in cerebral blood flow velocity in infants born at full term is caused by a simultaneous reduction in carbon dioxide tension that causes a comparatively large effect on the reduction of cerebral blood flow.

We thank Dr Kent Woods for statistical advice, and the nurses in the neonatal unit for their patience. DBS received financial support from the Spastics Society.

\section{References}

1 Dubowitz LMS, Dubowitz V, Goldberg C. Clinical assessment of gestational age in the newborn infant. J Pediatr 1970;77:1-10.

2 Schlindwein FS, Smith MJ, Evans DH. Spectral analysis of Doppler signals and computation of the normalised first moment in real-time using a digital signal processor. Med Biol Eng Comput 1988;26:228-32.

3 Gibbons DT, Evans DH, Barrie WW, Cosgriff PS. Real-time calculation of ultrasonic pulsatility index. Med Biol Eng Comput 1981;19:28-34.

4 Evans DH. On the measurement of the mean velocity of blood flow over the cardiac cycle using Doppler ultrasound. Ultrasound Med Biol 1985;11:735-41.

${ }^{5}$ Evans DH, Levene MI, Shortland DB, Archer LNJ. Resistance index, blood flow velocity, and resistance area product in the cerebral arteries of very low birth weight infants during the first week of life. Ultrasound Med Biol 1988;14:103-10.

${ }^{6}$ Levene MI, Shortland DB, Gibson N, Evans, DH. Carbon dioxide reactivity of the cerebral circulation in extremely premature infants: effects of postnatal age and indomethacin. Pediatr Res 1988 (in press).

7 Purves MJ, James IM. Observations on the control of cerebral blood flow in the sheep fetus and newborn lamb. Circ Res 1969;25:651-67.

${ }^{8}$ Rosenberg AA, Narayanan V, Jones MD. Comparison of anterior cerebral artery blood flow velocity and cerebral blood flow during hypoxia. Pediatr Res 1985;19:67-70. 
${ }^{9}$ Costeloe K, Smyth DPL, Murdoch N, Rolfe P, Tizard JPM. A comparison between electrical impedance and strain gauge plethysmography for the study of cerebral blood flow in the newborn. Pediatr Res 1984;18:290-5.

${ }^{10}$ Drayton MR, Skidmore R. Vasoactivity of the major intracranial arteries in newborn infants. Arch Dis Child 1987;62:236-40.

11 Mchedlishvili G. Physiological mechanisms controlling cerebral blood flow. Stroke 1980;11:240-8.

12 Mchedlishvili G. Regulation providing an adequate blood supply to cerebral tissue. In: Bevan JA. Arterial behaviour and blood circulation in the brain. New York: Plenum Press, 1986:96-164.
13 Shapiro W, Wassermann AJ, Patterson JL. Human cerebrovascular response to combined hypoxia and hypercapnia. Circ Res 1966;19:903-9.

14 Quint SR, Scremin OU, Sonnenschein RR, Rubinstein EH. Enhancement of cerebrovascular effect of $\mathrm{CO}_{2}$ by hypoxia. Stroke 1980;11:286-9.

Correspondence to Dr MI Levene, Department of Child Health, Leicester University School of Medicine, Leicester LE1 5WW.

Accepted 11 May 1988 